\title{
Accuracy of emergency medical service telephone triage of need for an ambulance response in suspected COVID-19: An observational cohort study
}

Carl Marincowitz ${ }^{1}$ NIHR Clinical Lecturer Emergency Medicine

Tony Stone ${ }^{1}$ Senior Data Manager

Madina Hasan ${ }^{1}$ Research Associate

Richard Campbell ${ }^{1}$ Data Specialist

Peter A. Bath ${ }^{1,2}$ Professor of Health Informatics and Health Data Analytics

Janette Turner ${ }^{1}$ Honorary Senior Research Fellow

Richard Pilbery ${ }^{3}$ Research paramedic

Benjamin Thomas ${ }^{4}$ Study Manager

Laura Sutton ${ }^{4}$ Research Associate

Fiona Bell ${ }^{3}$ Head of Research

Katie Biggs ${ }^{4}$ Research Fellow/Assistant CTRU Director

Frank Hopfgartner ${ }^{2}$ Senior Lecturer in Data Science

Suvodeep Mazumdar ${ }^{2}$ Lecturer in Data Analytics

Jennifer Petrie ${ }^{4}$ Trial Manager

Steve Goodacre ${ }^{1}$ Professor of Emergency Medicine

1. Centre for Urgent and Emergency Care Research (CURE), Health Services Research School of Health and Related Research, University of Sheffield, Regent Court, 30 Regent Street, Sheffield, S1 4DA, UK, Fax: +44 (0)114 2220749 Tel: (+44) (0)114 222 4345, Email: C.Marincowitz@Sheffield.ac.uk

2. Information School, University of Sheffield, Regent Court, 211 Portobello St, Sheffield S1 4DP, UK

3. Yorkshire Ambulance Service NHS Trust, Trust HQ, Springhill 2, Brindley Way, Wakefield, United Kingdom, WF2 OXQ

4. Clinical Trials Research Unit (CTRU), Health Services Research School of Health and Related Research, University of Sheffield, Regent Court, 30 Regent Street, Sheffield, S1 4DA, UK, Fax: +44 (0)114 2220749 Tel: (+44) (0)114 222 4345,

1 NOTE: This preprint reports new research that has not been certified by peer review and should not be used to guide clinical practice. 


\begin{abstract}
Background

Emergency Medical Services (EMS) have experienced surges in demand as the COVID-19 pandemic has progressed with ambulances services in the UK declaring major incidents due to the risk of care being compromised. COVID-19 specific EMS telephone triage tools have been introduced to help manage demand. There has been no previous evaluation of the accuracy of EMS telephone triage in identifying patients with suspected COVID-19 at risk of serious adverse outcome.
\end{abstract}

We aimed to assess accuracy of EMS telephone triage in identifying patients who need an EMS response and identify factors which affect triage accuracy.

Method

Patients who made an emergency call to Yorkshire Ambulance Service between $2^{\text {nd }}$ April and $29^{\text {th }}$ June 2020 and were assessed using an AMPDS pandemic pathway for suspected COVID-19 were linked to Office for National Statistics death registration data, hospital and general practice electronic health care data collected by NHS Digital.

Accuracy of decision to dispatch an ambulance was assessed in terms of death or need for organ support at 30 days from the first 999 call. Multivariable logistic regression was used to identify factors associated with false negative and false positive triage.

Results

Of 12,653 callers included in the study population, $11.1 \%$ experienced the primary composite adverse outcome. Using the triage pathway, $16 \%$ of callers did not receive an emergency response and they had a lower risk (3.5\%) of the primary outcome. Ambulances were dispatched to 4, 230 callers (33.4\%) who were not subsequently conveyed to hospital and did not experience the primary outcome (false positive triage). Multivariable modelling found older age and presence of pre-existing respiratory disease were significant predictors of false positive triage.

\title{
Conclusion
}

EMS telephone triage avoided $16 \%$ of calls receiving an emergency ambulance, of whom $3.5 \%$ died or needed organ support by 30 days. Telephone triage can therefore reduce the burden of EMS responses but with the cost of a small proportion of patients who do not receive an initial emergency response deteriorating. Research is needed to identify the appropriate balance between over- and under-triage 


\section{Background}

During the COVID-19 pandemic, emergency medical service (EMS) call volumes have been volatile in the UK, Europe and North America. Some ambulance services in the UK reported up to three times the expected number of EMS calls during the first and second waves of the pandemic, an increase also observed in other parts of Europe..$^{1-4}$ Other EMS providers in the UK and North America observed initial decreases in call volumes. ${ }^{5,6}$ Surges in demand in the second wave of the pandemic led to some ambulance services in the UK declaring major incidents and warning of care being compromised by overwhelming demand. ${ }^{7,8}$

EMS call handlers in the UK are typically trained non-clinical staff who use either the Advanced Medical Priority Dispatch System (AMPDS) or NHS Pathways to triage what emergency care response is needed to calls. ${ }^{4}$ On the $2^{\text {nd }}$ April 2020, six English NHS ambulance services using AMPDS introduced a specific protocol for callers with suspected COVID-19 using the pandemic Card 36. ${ }^{9,10}$ Structured questions (summarised in Supplementary Material 1) were used to triage urgency of EMS response into three levels: Delta (highest priority requiring immediate response), Charlie (medium priority requiring ambulance attendance when able) and Alpha (no ambulance dispatched). The AMPDS Card 36 was implemented by UK ambulance services in different ways, with some symptoms and selected high risk-groups receiving a higher priority response than normal, with alterations to the card implemented in the following months. ${ }^{4}$

There is evidence that changes in EMS practice due to the pandemic and delays in the emergency assessment of patients with COVID-19 may have contributed to avoidable deaths in the North West of England. ${ }^{11}$ Despite the need for EMS telephone triage to balance ambulance responses to patients requiring life-saving interventions against an anticipation of overwhelming demand during the pandemic, there has been no previous evaluation of the accuracy of the clinical risk-assessment performed for patients with suspected COVID-19.

Our study aimed to:

1) Assess how accurately EMS telephone triage identified those likely to suffer an adverse outcome and so need an emergency ambulance response.

2) Identify any factors that may have affected the accuracy of EMS telephone triage.

\section{Methods}

Study Design and Setting 
This observational cohort study used linked routinely collected EMS telephone call centre data from Yorkshire Ambulance Service NHS Trust (YAS) to assess the accuracy of clinical triage of patients with suspected COVID-19.

Emergency services provided by YAS covers a region in the north of England of approximately 6,000 square miles and with a population of 5.3 million. In 2020/2021, YAS received more than 1,000,000 emergency (999) calls.

\section{Data Sources and linkage}

Yorkshire Ambulance Service provided a dataset of all 999 calls, triaged using a Card 36 pandemic triage pathway for patients with suspected COVID-19, received between the $2^{\text {nd } A p r i l ~ a n d ~ 29 t h ~ J u n e ~}$ 2020. The dataset consisted of patient identifiers, demographic data, call details and the outcome of the call (including whether an ambulance was dispatched) extracted from routinely collected electronic call records.

Health and social care data relating to the population in England within the UK National Health Service (NHS) are managed by NHS Digital. We provided patient identifiers to NHS Digital to trace patients in our cohort and supply additional individual-level demographic, co-morbidity and outcome data. NHS Digital identified records in their collections belonging to patients in our cohort, and provided data on patient demographics, limited COVID-related general practice (GP) records, emergency department attendances, hospital inpatient admissions, critical care periods, and death registrations from the UK Office for National Statistics (ONS).

YAS and NHS Digital removed records where patients indicated that they did not wish their data to be used for research purposes, via the NHS data opt-out service. ${ }^{12}$ The study team also excluded patients who had opted out of any part of the wider PRIEST study ${ }^{2}$, of which this evaluation forms a part, and those with inconsistent records (e.g., multiple deaths recorded or death before latest activity). Patient identifiers across all datasets were replaced with a consistent pseudo-identifier to enable the identification and linkage of records belonging to the same patient across all datasets but without revealing any patient identifiers. Calls which originated from other health care services (i.e., where a decision that ambulance dispatch was required had already been made) were excluded from the study population.

Inclusion criteria

Our final cohort consisted of all adult (aged 16 years and over) callers at time of first (index) EMS 999 call between 2nd April and 29th June 2020 assessed using the suspected COVID-19 Card 36 triage pathway, who were successfully traced by NHS Digital. 


\section{Outcome}

The primary outcome was death, renal, respiratory, or cardiovascular organ support (identified from death registration and critical care data) at 30 days from index contact.

The secondary outcomes were hospital conveyance following ambulance dispatch (transfer to ED or inpatient setting) and inpatient admission (recoded inpatient dataset) 30 days from index contact.

\section{Patient Characteristics}

Consistent with methods used to estimate the Charlson comorbidity index from the available routine data, comorbidities were included if recorded within 12 months before the index EMS call. ${ }^{13,14}$ In a similar way, only immunosuppressant drug prescriptions documented in GP records within 30 days before the index contact, contributed to the immunosuppression co-morbidity variable. Pregnancy status was based on GP records recorded in the previous 9 months. Frailty in patients older than 65 years was derived from the latest recorded Clinical Frailty Scale (CFS) score (if recorded) in the electronic GP records prior to index attendance. ${ }^{15}$ Patients under the age of 65 years were not given a CFS score since it is not validated in this age group. However, in multivariable analysis, patients under the age of 65 were assumed to have a functional level equivalent to a mild frailty category (CFS 1-3).

\section{Analysis}

We conducted a descriptive analysis of caller demographics, co-morbidities and call disposition. The proportion of callers who experienced the primary and secondary outcome was estimated. To assess the accuracy of EMS telephone triage in identifying clinical outcomes requiring an emergency response, the call disposition of the index contact was divided into the binary classification of either: ambulance dispatched (Delta or Charlie priority); or other call outcome (Alpha priority) (Supplementary Material 1). We assessed the accuracy of the binary triage classification (ambulance dispatched versus no ambulance dispatched) in terms of sensitivity, specificity, positive predictive value (PPV) and negative predictive value (NPV) for the primary and secondary outcomes with $95 \%$ confidence intervals $(\mathrm{Cl})$.

Patient characteristics of false negatives (those who experienced the primary outcome and no ambulance was dispatched) and true positives (ambulance dispatched who experienced the primary outcome) were compared. In patients with the adverse outcome multi-variable logistic regression was used to identify patient characteristics associated with false negative triage. We also compared 
the characteristics of false positives (ambulance dispatched and not conveyed to hospital) and true negatives (no ambulance dispatched) among those who did not experience the primary composite outcome. We used multivariable logistic regression to identify factors which predicted false positive EMS triage. Obesity was excluded from multivariable analysis due to an observed implausible protective association with the primary outcome, which we believe to be an artefact of how these data were collected and recorded in the electronic GP data set. Ethnicity and frailty were also excluded from multivariable analysis due to the high proportion of missing data. All analyses were completed using STATA 16 (StataCorp. 2019. Stata Statistical Software: Release 16. College Station, TX: StataCorp LLC).

\section{Ethical Approval}

The North West-Haydock Research Ethics Committee gave a favourable opinion on the PAINTED study on 25 June 2012 (reference 12/NW/0303) and on the updated PRIEST study on 23rd March 2020, including the analyses presented here. The Confidentiality Advisory Group of the NHS Health Research Authority granted approval to collect data without patient consent in line with Section 251 of the National Health Service Act 2006. Access to data collected by NHS Digital was recommended for approval by its Independent Group Advising on the Release of Data (IGARD) on $11^{\text {th }}$ September 2021, having received additional recommendation for approval for access to GP records from the Profession Advisory Group (PAG) on $19^{\text {th }}$ August 2021.

\section{Patient Public Involvement}

The Sheffield Emergency Care Forum (SECF) is a public representative group interested in emergency care research. ${ }^{16}$ Members of SECF advised on the development of the PRIEST study and two members joined the Study Steering Committee. Patients were not involved in the conduct of the study.

Results

All totals (including outcome) presented are rounded to the nearest 5, with small numbers suppressed to comply with NHS Digital data disclosure guidance. ${ }^{17}$

Study population 
medRxiv preprint doi: https://doi.org/10.1101/2021.10.12.21264136; this version posted October 19, 2021. The copyright holder for this preprint (which was not certified by peer review) is the author/funder, who has granted medRxiv a license to display the preprint in perpetuity.

It is made available under a CC-BY 4.0 International license .

Figure 1 and Table 1 summarise study cohort derivation and the characteristics of the 12, 653 included individual callers. In total, 1,405 callers $(11.1 \%, 95 \% \mathrm{Cl}: 10.5$ to $11.7 \%)$ experienced the primary outcome (death or organ support) within 30 days following the index EMS 999 call. An ambulance was dispatched to $10,650(84.2 \%)$ of callers. In our study cohort, 6,070 patients $(48 \%$. 95\%: 47.1 to $48.9 \%$ ) were conveyed to hospital (ED or directly to an inpatient setting) and 5,735 (45.3\%, 95\% Cl: 44.5 to $46.2 \%$ ) were admitted as hospital in patients within 30 days of index contact.

The median age of the whole cohort was 66 (IQR $=46-81)$, the cohort had an almost equal proportion of males $(49.5 \%)$ and females $(50.5 \%)$ and had high rates of comorbidity (chronic respiratory disease $29.5 \%$, diabetes $16.6 \%$ and hypertension $35.9 \%$ ).

Table 1: Population characteristics

\begin{tabular}{|c|c|c|c|}
\hline $\begin{array}{c}\text { Population } \\
\text { Characteristic }\end{array}$ & Level & $\begin{array}{l}\text { Whole Population } \\
\qquad \mathrm{N}=12,653\end{array}$ & $\begin{array}{c}\text { Adverse Outcome (30 } \\
\text { days) } N=1,405\end{array}$ \\
\hline $\begin{array}{c}\text { Age } \\
\text { (Years) }\end{array}$ & $\begin{array}{l}\text { Median (IQR)* } \\
\text { Mean }\end{array}$ & $\begin{array}{c}66(46-81) \\
62.3\end{array}$ & $\begin{array}{c}80(67-87) \\
70\end{array}$ \\
\hline $\operatorname{Sex}(N, \%)$ & Male & $\begin{array}{c}6,260 \\
(49.5 \%)\end{array}$ & $\begin{array}{c}795 \\
(56.5 \%)\end{array}$ \\
\hline \multirow[t]{10}{*}{ Comorbidity (N, \%) } & Cardiovascular Disease & $\begin{array}{c}810 \\
(6.4 \%)\end{array}$ & $\begin{array}{c}120 \\
(8.6 \%)\end{array}$ \\
\hline & Chronic Resp. Disease & $\begin{array}{c}3,730 \\
(29.5 \%) \\
\end{array}$ & $\begin{array}{c}410 \\
(29.4 \%) \\
\end{array}$ \\
\hline & Diabetes & $\begin{array}{c}2,095 \\
(16.5 \%)\end{array}$ & $\begin{array}{c}325 \\
(23.1 \%)\end{array}$ \\
\hline & Hypertension & $\begin{array}{c}4,545 \\
(35.9 \%)\end{array}$ & $\begin{array}{c}705 \\
(50.4 \%) \\
\end{array}$ \\
\hline & $\begin{array}{l}\text { Immunosuppression } \\
\text { (including steroid use) }\end{array}$ & $\begin{array}{c}2,540 \\
(20.1 \%)\end{array}$ & $\begin{array}{c}360 \\
(25.8 \%)\end{array}$ \\
\hline & Active Malignancy & $\begin{array}{c}595 \\
(4.7 \%) \\
\end{array}$ & $\begin{array}{c}160 \\
(11.5 \%)\end{array}$ \\
\hline & Obesity & $\begin{array}{c}1,960 \\
(15.5 \%)\end{array}$ & $\begin{array}{c}100 \\
(7.3 \%)\end{array}$ \\
\hline & Pregnant & $\begin{array}{c}135 \\
(1.1 \%) \\
\end{array}$ & $*$ \\
\hline & Renal Impairment & $\begin{array}{c}365 \\
(2.9 \%)\end{array}$ & $\begin{array}{c}60 \\
(4.3 \%)\end{array}$ \\
\hline & Stroke & $\begin{array}{c}285 \\
(2.2 \%) \\
\end{array}$ & $\begin{array}{c}45 \\
(3.1 \%) \\
\end{array}$ \\
\hline Social (N, \%) & Smoker & $4,810(38.0 \%)$ & $550(39.3 \%)$ \\
\hline \multirow{4}{*}{$\begin{array}{c}\text { Number of Prescribed } \\
\text { Drugs Used } \\
(\mathrm{N}, \%)\end{array}$} & 0 & $3,115(24.6 \%)$ & $175(12.4 \%)$ \\
\hline & $1-5$ & $6,015(47.5 \%)$ & $700(49.8 \%)$ \\
\hline & $6-10$ & $3,030(23.9 \%)$ & $465(33.1 \%)$ \\
\hline & 11 or more & $490(3.9 \%)$ & $65(4.7 \%)$ \\
\hline \multirow{2}{*}{$\begin{array}{l}\text { Clinical Frailty Scale } \\
\qquad(\mathrm{N}, \%)\end{array}$} & Unknown & $4,560(36.0 \%)$ & $685(48.9 \%)$ \\
\hline & Aged $<65$ & $6,105(48.2 \%)$ & $305(21.6 \%)$ \\
\hline
\end{tabular}


medRxiv preprint doi: https://doi.org/10.1101/2021.10.12.21264136; this version posted October 19, 2021. The copyright holder for this preprint (which was not certified by peer review) is the author/funder, who has granted medRxiv a license to display the preprint in perpetuity.

It is made available under a CC-BY 4.0 International license .

\begin{tabular}{|c|c|c|c|}
\hline & $1-3$ & $165(1.3 \%)$ & $15(1.0 \%)$ \\
\hline & $3-6$ & $670(5.3 \%)$ & $85(6.0 \%)$ \\
\hline & $6-9$ & $1,155(9.1 \%)$ & $315(22.5 \%)$ \\
\hline \multirow[t]{6}{*}{ Ethnicity (N, \%) } & Asian or Asian British & $750(5.9 \%)$ & $5(3.6 \%)$ \\
\hline & Black or Black British & $190(1.5 \%)$ & $15(0.9 \%)$ \\
\hline & Mixed & $110(0.9 \%)$ & $*$ \\
\hline & Other Ethnic Groups & $160(1.3 \%)$ & $*$ \\
\hline & White & $9,020(71.3 \%)$ & $950(67.8 \%)$ \\
\hline & Unknown & $2,425(19.2 \%)$ & $375(26.6 \%)$ \\
\hline \multirow{6}{*}{$\begin{array}{c}\text { Deprivation Index (N, } \\
\%)\end{array}$} & Unknown & $1,250(9.9 \%)$ & $190(13.5 \%)$ \\
\hline & $1-2$ & $4,600(40.3 \%)$ & $440(36.4 \%)$ \\
\hline & $3-4$ & $2,075(18.2 \%)$ & $220(18 \%)$ \\
\hline & $5-6$ & $1,880(16.5 \%)$ & $220(18 \%)$ \\
\hline & $7-8$ & $1,695(14.9 \%)$ & $210(17.2 \%)$ \\
\hline & $9-10$ & $1,160(10.2 \%)$ & $130(10.6 \%)$ \\
\hline \multirow{2}{*}{$\begin{array}{l}\text { Ambulance Dispatched } \\
\text { (N, \%) }\end{array}$} & Ambulance & $10,650(84.2 \%)$ & $1,335(95 \%)$ \\
\hline & No Ambulance & $2,000(15.8 \%)$ & $70(5 \%)$ \\
\hline \multirow[t]{3}{*}{ Outcome (N, \%) } & Death & $\begin{array}{c}1,155 \\
(9.1 \%) \\
\end{array}$ & $\begin{array}{c}1,155 \\
(82.4 \%) \\
\end{array}$ \\
\hline & $\begin{array}{l}\text { Deaths due to COVID } \\
\text { (including after } 30 \text { days) }\end{array}$ & $690(5.5 \%)$ & $530(37.8 \%)$ \\
\hline & $\begin{array}{c}\text { Organ support } \\
\text { (within } 30 \text { days) }\end{array}$ & $\begin{array}{c}335 \\
(2.6 \%)\end{array}$ & $\begin{array}{c}335 \\
(23.8 \%)\end{array}$ \\
\hline \multirow[t]{2}{*}{ Hospitalisation (N, \%) } & ED Attendance & $\begin{array}{c}6,945 \\
(54.9 \%)\end{array}$ & $\begin{array}{c}970 \\
(69.1 \%)\end{array}$ \\
\hline & Inpatient admission & $\begin{array}{c}5,735 \\
(45.3 \%)\end{array}$ & $\begin{array}{c}1,085 \\
(77.3 \%)\end{array}$ \\
\hline $\begin{array}{c}\text { Confirmed Hospital } \\
\text { Diagnosis of COVID (N, } \\
\%)^{* *}\end{array}$ & $\begin{array}{c}\text { In ED or as inpatient at } \\
30 \text { days }\end{array}$ & $\begin{array}{l}1,895 \\
(15 \%)\end{array}$ & $\begin{array}{c}595 \\
(42.3 \%)\end{array}$ \\
\hline \multirow{2}{*}{$\begin{array}{l}\text { Time to Primary } \\
\text { Outcome from index } \\
\text { contact- up to and } \\
\text { including }(\mathrm{N}, \%)\end{array}$} & 72 hours & $475(3.8 \%)$ & $475(33.6 \%)$ \\
\hline & 7 days & $780(6.1 \%)$ & $780(55.5 \%)$ \\
\hline
\end{tabular}

*Interquartile Range (IQR)

**Unrestricted community testing for suspected COVID-19 was only available from the 18/05/2020. Confirmed diagnosis is based upon inpatient PCR testing or clinical diagnosis in hospital

Accuracy of telephone triage of ambulance dispatch

Table 2 shows the accuracy of the EMS telephone triage decision to dispatch an ambulance for the composite primary outcome indicating need for emergency intervention. Decision to dispatch an ambulance achieved a sensitivity of $95 \%$ (95\% $\mathrm{Cl}: 93.7$ to $96.1 \%)$ to the primary outcome. If advised 
medRxiv preprint doi: https://doi.org/10.1101/2021.10.12.21264136; this version posted October 19, 2021. The copyright holder for this preprint (which was not certified by peer review) is the author/funder, who has granted medRxiv a license to display the preprint in perpetuity.

It is made available under a CC-BY 4.0 International license .

to self-care/non-urgent clinical assessment, the chance of experiencing an adverse outcome was approximately $3.5 \%$ (NPV: $96.5 \%, 95 \% \mathrm{Cl}: 95.6$ to $97.2 \%$ ). The high sensitivity was achieved at the cost of specificity (17.2\% $95 \% \mathrm{Cl}: 16.5 \%$ to $17.9 \%)$. Among patients for whom an ambulance was dispatched, the risk of serious adverse outcomes was $12.5 \%$ (95\% Cl: 11.9 to $13.2 \%)$, transfer to hospital was 57\% (95\% Cl: 56 to 58\%) and hospital admission $49.3 \%$ (48.3 to 50.2\%).

Table 2: Performance of decision to dispatch ambulance for

i) composite primary outcome (death or organ support)

\begin{tabular}{|l|l|l|l|}
\hline \multicolumn{3}{|c|}{ Adverse outcome 30 days (11.1\%, 10.5-11.7\%) } \\
\hline $\mathrm{N}=12,653$ & Adverse Outcome & $\begin{array}{l}\text { No Adverse } \\
\text { Outcome }\end{array}$ & \\
\hline Ambulance dispatched & 1,335 & 9,320 & $\begin{array}{l}\text { Sensitivity 95\% (93.7- } \\
96.1 \%) \\
\text { Positive Predictive Value } \\
12.5 \%(11.9-13.2 \%)\end{array}$ \\
\hline No Ambulance dispatched & 70 & & $\begin{array}{l}\text { Specificity 17.2\% (16.5\% - } \\
17.9 \%) \\
\end{array}$ \\
& & 1,930 & $\begin{array}{l}\text { Negative Predictive Value } \\
\text { 96.5\% (95.6 - 97.2\%) }\end{array}$ \\
\hline
\end{tabular}

Prediction of false negative or false positive ambulance dispatch

Table 3 compares the characteristics of patients who experienced the primary outcome, and either did (true positives) or did not (false negatives) have an ambulance dispatched on index call. In both groups, over $50 \%$ of people experienced the primary adverse outcome within seven days of first contact and around $33 \%$ of patients experienced the adverse outcome within 72 hours of index assessment. Multivariable modelling (Supplementary Material 2) showed that female sex (OR 1.89, $95 \% \mathrm{Cl} 1.09$ to 3.26 ) was associated with increased risk and, increasing age (OR $0.95,95 \% \mathrm{Cl} 0.94$ to 0.97 ) and malignancy (OR $0.12,95 \% \mathrm{Cl} 0.02$ to 0.92 ) reduced risk, of false negative triage.

Table 3: False negatives (No ambulance dispatch and adverse outcome) compared to true positives (ambulance dispatch and adverse outcome)

\begin{tabular}{|c|c|c|c|}
\hline $\begin{array}{c}\text { Population } \\
\text { Characteristic }\end{array}$ & Level & $\begin{array}{c}\text { False Negatives (30 } \\
\text { days) } \mathbf{N = 7 0}\end{array}$ & $\begin{array}{c}\text { True Positives (30 } \\
\text { days) } \mathbf{N = 1 , 3 3 5}\end{array}$ \\
\hline Age & Median (IQR) & $61.5(51-80)$ & $80(68-87)$ \\
(Years) & Mean & 64 & 76.7 \\
\hline Sex (N, \%) & Male & 35 & 760 \\
& & $(51.4 \%)$ & $(56.8 \%)$ \\
\hline
\end{tabular}


medRxiv preprint doi: https://doi.org/10.1101/2021.10.12.21264136; this version posted October 19, 2021. The copyright holder for this preprint (which was not certified by peer review) is the author/funder, who has granted medRxiv a license to display the preprint in perpetuity.

It is made available under a CC-BY 4.0 International license .

\begin{tabular}{|c|c|c|c|}
\hline \multirow[t]{10}{*}{ Comorbidity (N, \%) } & Cardiovascular Disease & $*$ & $115(8.8 \%)$ \\
\hline & Chronic Resp. Disease & $15(20 \%)$ & $400(29.9 \%)$ \\
\hline & Diabetes & $20(25.7 \%)$ & $305(23 \%)$ \\
\hline & Hypertension & $25(34.3 \%)$ & $685(51.2 \%)$ \\
\hline & $\begin{array}{l}\text { Immunosuppression } \\
\text { (including steroid use) }\end{array}$ & $10(17.1 \%)$ & $350(26.3 \%)$ \\
\hline & Active Malignancy & $*$ & $160(11.9 \%)$ \\
\hline & Obesity & $10(12.9 \%)$ & $95(7 \%)$ \\
\hline & Pregnant & $*$ & $*$ \\
\hline & Renal Impairment & $*$ & $55(4.2 \%)$ \\
\hline & Stroke & $*$ & $40(3.2 \%)$ \\
\hline Social (N, \%) & Smoker & $25(32.9 \%)$ & $530(39.6 \%)$ \\
\hline \multirow{4}{*}{$\begin{array}{l}\text { Number of Drugs Used } \\
\qquad(\mathrm{N}, \%)\end{array}$} & 0 & $20(25.7 \%)$ & $155(11.7 \%)$ \\
\hline & $1-5$ & $30(42.9 \%)$ & $670(50.2 \%)$ \\
\hline & $6-10$ & $20(28.6 \%)$ & $445(33.3 \%)$ \\
\hline & 11 or more & $*$ & $65(4.8 \%)$ \\
\hline \multirow{5}{*}{$\begin{array}{l}\text { Clinical Frailty Scale } \\
\qquad(\mathrm{N}, \%)\end{array}$} & Unknown & $60(84.3 \%)$ & $665(49.8 \%)$ \\
\hline & Aged $<65$ & $*$ & $260(19.7 \%)$ \\
\hline & $1-3$ & $*$ & $15(1 \%)$ \\
\hline & $3-6$ & $*$ & $80(6.2 \%)$ \\
\hline & $6-9$ & $*$ & $310(23.4 \%)$ \\
\hline \multirow[t]{6}{*}{ Ethnicity (N, \%) } & Asian or Asian British & * & $45(3.5 \%)$ \\
\hline & Black or Black British & $*$ & $15(1 \%)$ \\
\hline & Mixed & $*$ & $*$ \\
\hline & Other Ethnic Groups & $*$ & $10(0.7 \%)$ \\
\hline & White & $45(61.4 \%)$ & $910(68.1 \%)$ \\
\hline & Unknown & $20(28.6 \%)$ & $355(26.5 \%)$ \\
\hline \multirow{6}{*}{$\begin{array}{c}\text { Deprivation Index (N, } \\
\%)\end{array}$} & Unknown & $*$ & $185(13.7 \%)$ \\
\hline & $1-2$ & $30(46 \%)$ & $410(35.8 \%)$ \\
\hline & $3-4$ & $10(17.4 \%)$ & $205(17.9 \%)$ \\
\hline & $5-6$ & $*$ & $210(18.4 \%)$ \\
\hline & $7-8$ & $10(17.4 \%)$ & $200(17.2 \%)$ \\
\hline & $9-10$ & $*$ & $120(10.6 \%)$ \\
\hline \multirow[t]{3}{*}{ Outcome (N, \%) } & Death & $45(65.7 \%)$ & $1,110(83.3 \%)$ \\
\hline & $\begin{array}{c}\text { Deaths due to COVID } \\
\text { (including after } 30 \\
\text { days) }\end{array}$ & $20(27.2 \%)$ & $520(39 \%)$ \\
\hline & $\begin{array}{c}\text { Organ support } \\
\text { (within } 30 \text { days) }\end{array}$ & $\begin{array}{c}35 \\
(47.1 \%) \\
\end{array}$ & $\begin{array}{c}300 \\
(22.6 \%)\end{array}$ \\
\hline \multirow[t]{2}{*}{ Hospitalisation (N, \%) } & ED attendance & $\begin{array}{c}45 \\
(65.7 \%) \\
\end{array}$ & $\begin{array}{c}925 \\
(69.2 \%) \\
\end{array}$ \\
\hline & Inpatient admission & $\begin{array}{c}50 \\
(72.9 \%) \\
\end{array}$ & $\begin{array}{c}1,035 \\
(77.6 \%) \\
\end{array}$ \\
\hline $\begin{array}{c}\text { Confirmed Hospital } \\
\text { Diagnosis of COVID (N, } \\
\%)^{* *}\end{array}$ & $\begin{array}{l}\text { In ED or as inpatient at } \\
30 \text { days }\end{array}$ & $25(34.3 \%)$ & $570(42.7 \%)$ \\
\hline \multirow{2}{*}{$\begin{array}{l}\text { Time to Primary } \\
\text { Outcome from index } \\
\text { contact- up to and } \\
\text { including }(\mathrm{N}, \%)\end{array}$} & 72 hours & $25(37.1 \%)$ & $445(33.5 \%)$ \\
\hline & 7 days & $45(62.8 \%)$ & $730(54.9 \%)$ \\
\hline
\end{tabular}


Table 4 compares the characteristics of patients without the primary outcome for whom an ambulance was dispatched and the patient was not transferred to hospital (False Positives), or no ambulance was dispatched (True Negatives). $33.4 \%$ of the cohort were false positives and Supplementary Material 3 presents the results of multivariable modelling to identify factors associated with false postivie triage. Risk of false positive was strongly associated with chronic respiratory disease (OR $1.3595 \% \mathrm{Cl} 1.13$ to 1.60 ) and reduced in smokers (OR $0.86,95 \% \mathrm{Cl} 0.75$ to 0.99). Increasing age, deprivation and female sex were also associated with risk of false positive triage.

Table 4: False positive (Ambulance dispatched, not conveyed to hospital and no adverse outcome) compared to true negatives

\begin{tabular}{|c|c|c|c|}
\hline $\begin{array}{c}\text { Population } \\
\text { Characteristic }\end{array}$ & Level & $\begin{array}{c}\text { False Positive (30 } \\
\text { days) } N=4,230\end{array}$ & $\begin{array}{c}\text { True Negative (30 } \\
\text { days) } N=1,930\end{array}$ \\
\hline $\begin{array}{c}\text { Age } \\
\text { (Years) }\end{array}$ & $\begin{array}{l}\text { Median (IQR) } \\
\text { Mean }\end{array}$ & $\begin{array}{c}66(46-81) \\
62.9\end{array}$ & $\begin{array}{c}37(28-53) \\
41.6\end{array}$ \\
\hline $\operatorname{Sex}(N, \%)$ & Male & $1,920(45.4 \%)$ & $1000(51.7 \%)$ \\
\hline \multirow[t]{10}{*}{ Comorbidity (N, \%) } & $\begin{array}{c}\text { Cardiovascular } \\
\text { Disease }\end{array}$ & $\begin{array}{c}235 \\
(5.5 \%) \\
\end{array}$ & $\begin{array}{c}30 \\
(1.6 \%) \\
\end{array}$ \\
\hline & $\begin{array}{l}\text { Chronic Resp. } \\
\text { Disease }\end{array}$ & $\begin{array}{c}1,280 \\
(30.3 \%)\end{array}$ & $\begin{array}{c}380 \\
(19.5 \%)\end{array}$ \\
\hline & Diabetes & $600(14.2 \%)$ & $155(7.8 \%$ \\
\hline & Hypertension & $1,480(35 \%)$ & $255(13.1 \%)$ \\
\hline & $\begin{array}{c}\text { Immunosuppression } \\
\text { (including steroid } \\
\text { use) }\end{array}$ & $\begin{array}{c}825 \\
(19.5 \%)\end{array}$ & $\begin{array}{c}180 \\
(9.4 \%)\end{array}$ \\
\hline & Active Malignancy & $140(3.3 \%)$ & $20(0.9 \%)$ \\
\hline & Obesity & $700(16.5 \%)$ & $260(13.4 \%)$ \\
\hline & Pregnant & $50(1.2 \%)$ & $40(2.2 \%)$ \\
\hline & Renal Impairment & $110(2.6 \%)$ & $15(0.8 \%)$ \\
\hline & Stroke & $90(2.1 \%)$ & $15(0.7 \%)$ \\
\hline Smoking Status (N, \%) & Smoker & $1,570(37.1 \%)$ & $640(33.2 \%)$ \\
\hline \multirow{4}{*}{$\begin{array}{l}\text { Number of Drugs Used } \\
\qquad(\mathrm{N}, \%)\end{array}$} & 0 & $1,040(24.6 \%)$ & $955(49.3 \%)$ \\
\hline & $1-5$ & $2,075(49 \%)$ & $805(41.7 \%)$ \\
\hline & $6-10$ & $970(22.9 \%)$ & $150(7.6 \%)$ \\
\hline & 11 or more & $150(3.5 \%)$ & $25(1.4 \%)$ \\
\hline \multirow{5}{*}{$\begin{array}{l}\text { Clinical Frailty Scale } \\
(\mathrm{N}, \%)\end{array}$} & Unknown & $1,520(35.9 \%)$ & $150(7.6 \%)$ \\
\hline & Aged $<65$ & $2,030(48 \%)$ & $1,735(89.8 \%)$ \\
\hline & $1-3$ & $55(1.3 \%)$ & $*$ \\
\hline & $3-6$ & $245(5.7 \%)$ & $25(1.2 \%)$ \\
\hline & $6-9$ & 385 (9.1\%) & $25(1.2 \%)$ \\
\hline \multirow[t]{3}{*}{ Ethnicity (N, \%) } & Asian or Asian British & $250(5.9 \%)$ & $180(9.2 \%)$ \\
\hline & Black or Black British & $60(1.4 \%)$ & $50(2.5 \%)$ \\
\hline & Mixed & $35(0.9 \%)$ & $30(1.4 \%)$ \\
\hline
\end{tabular}




\begin{tabular}{|c|c|c|c|}
\hline \multirow{4}{*}{$\begin{array}{c}\text { Deprivation Index (N) } \\
\text { \%) }\end{array}$} & Other Ethnic Groups & $50(1.2 \%)$ & $65(3.3 \%)$ \\
\cline { 2 - 4 } & White & $3,080(72.7 \%)$ & $1,210(62.7 \%)$ \\
\cline { 2 - 4 } & Unknown & $760(17.9 \%)$ & $405(20.9 \%)$ \\
\cline { 2 - 4 } & Unknown & $420(10 \%)$ & $130(6.6 \%)$ \\
\cline { 2 - 4 } & $1-2$ & $1,470(38.6 \%)$ & $925(51.3 \%)$ \\
\cline { 2 - 4 } & $3-4$ & $720(18.9 \%)$ & $320(17.7 \%)$ \\
\cline { 2 - 4 } & $5-6$ & $565(17.2 \%)$ & $190(10.5 \%)$ \\
\hline
\end{tabular}

\section{Discussion}

Summary

Our study showed that callers with suspected COVID-19 who made an EMS call had a high rate of adverse outcomes (11.1\%, $95 \% \mathrm{Cl}: 10.5$ to $11.7 \%$ ). This is around four times greater than the adverse outcome rate in callers who contacted the NHS 111 telephone service and half that seen in an ED population with suspected COVID-19. ${ }^{18,19}$ Ambulances were dispatched to the majority of callers (84.2\%) and the decision to dispatch an ambulance provided good, but not excellent, prediction for adverse outcomes (sensitivity $95 \%, 95 \% \mathrm{Cl}: 93.7$ to $96.1 \%$ ). Callers for whom an ambulance was not dispatched had a $3.5 \%$ (NPP 96.5\% 95\% Cl: 95.6 to $97.2 \%$ ) risk of the primary composite adverse outcome. Our evaluation cannot account for instances where ambulance dispatch was not clinically appropriate despite a high-risk of significant adverse outcomes (e.g. transfer of care to community services for palliative care).

The cost of the high sensitivity achieved by dispatching ambulances to $84 \%$ of callers was a low specificity to the primary outcome (17.2\% $95 \% \mathrm{Cl}$ : $16.5 \%$ to $17.9 \%)$. In total, $33.4 \%$ of the cohort had an ambulance dispatched, and were not subsequently conveyed to hospital and nor did they experience the primary outcome. We used multivariable analysis to identify predictors of false negative and false positive triage. The findings need cautious interpretation, given the limited information available during telephone triage, but suggest that some co-morbidities (such as chronic respiratory disease) and increasing age may be over-estimated as predictors of adverse outcome.

\section{Strengths and limitations}

Although specific EMS telephone triage protocols have been introduced for patients with suspected COVID-19, this appears to be the first evaluation of triage accuracy. ${ }^{4}$ Our study used a large cohort of patients identified from routinely collected EMS records and linked this to nationally collected, patient-level healthcare data to provide robust clinical outcomes. We have assessed performance in a cohort of patients with suspected infection which, in the absence of accurate universally available 
rapid COVID-19 diagnostic tests, reflects the population who must be clinically triaged by urgent and emergency care services.

We have evaluated the performance EMS telephone triage for patients with suspected COVID-19 implemented by the Yorkshire Ambulance Service NHS Trust. Although use of the Card 36 protocol was recommended nationally, there was variation in implementation between different ambulance services. ${ }^{4}$ Our study used data from the first wave of the pandemic and it was not until later waves that some ambulance services came under significant pressures due to increased demand. ${ }^{1}$

Differences in EMS telephone triage demand and population characteristics of callers with suspected COVID-19 in later waves of the pandemic may affect the estimated accuracy of EMS decision to dispatch an ambulance. The implementation of a senior clinical support model within the call centre, and the influence of this secondary triage both of incoming calls and to support on-scene ambulance decision making was not quantified in this study. It was not possible to identify within the dataset which of the cohort were reviewed by a senior clinician at the initial call.

Our study used routinely collected data and consequently there were high rates of missing data for some variables, such as ethnicity and frailty. This prevented inclusion of these and non-routinely collected variables in analysis. We have assumed that if comorbidities or medication use was not recorded, they were not present. The mechanism of how data are collected and recorded in the routine data sets used means that there may be bias in the classification of patients. We have previously identified this for the obesity variable where the estimated prevalence of obesity in our cohort is $15 \%$ (half that reported in the national health survey) and an implausible protective association with adverse outcomes was observed. ${ }^{18,20}$ As weight is not comprehensively and consistently measured by GPs, estimated statistical effects may reflect unknown characteristics associated with a measurement being taken, rather than the variable itself.

Implications

EMS telephone triage of ambulance dispatch achieved a higher sensitivity for adverse outcomes than triage methods used for patient acuity in the ED and NHS-111 COVID-19 assessment pathways. ${ }^{21,22}$ Our cohort had a baseline risk of $11.1 \%$ of the primary outcome and serious adverse outcomes. The EMS telephone triage system selected those with a lower, 3.5\% risk of the primary outcome, not to have an ambulance dispatched. Given the high baseline risk, an alternative would have been to dispatch an ambulance to every caller. This would have led to around 2000 more ambulance being dispatched within the time-period (an 18.8\% increase). However, even using EMS telephone triage of ambulance dispatch, around a third of ambulances were dispatched to callers who were not subsequently conveyed to hospital and did not experience the primary outcome. In 
later waves of the pandemic, EMS providers in the UK experienced significant increases in demand with several ambulance services declaring major incidents. ${ }^{8,23,24}$ The accuracy of triage observed in our cohort may be the best that realistically can be achieved given the limited information available with telephone triage. However, any measures which can increase accuracy of EMS telephone triage, especially in terms of increasing specificity to adverse outcomes, without reducing sensitivity, are greatly needed.

Our exploratory multivariable analysis indicates that older age and presence of pre-existing respiratory disease were associated with a higher rate of false positive triage among those who did not have an adverse event, and therefore may be overestimated in the triage process as predictors of adverse outcomes. Being aged 65 years or over is included as a specific high-risk factor in the Card 36 pandemic triage tool. ${ }^{25}$ Use of a higher age threshold, or age in conjunction with variables related to performance status, could improve the specificity of EMS ambulance dispatch to significant adverse outcomes. ${ }^{26}$ Vaccination against COVID-19 or previous infection may also act to reduce the risk of serious adverse outcomes in suspected infection and these factors are not included in Card 36 assessment. ${ }^{27}$

Limited information is available during EMS telephone triage determining whether ambulance dispatch is required. The Card 36 pandemic triage tool used for this decision making in patients with suspected COVID-19 is consensus based. Empirical research assessing the predictive effect of all available variables is required if accuracy of triage, especially in terms of current over-triage, is to be improved. Given the limitations of the available routine data we have identified in this study, this is likely to require robust prospective data collection. The current high levels of demand that ambulance services are experiencing mean that even small gains in specificity which do not compromise sensitivity could have a large positive effect.

Conclusion

EMS telephone triage of need for ambulance dispatch in the first wave of the pandemic identified a population to whom ambulances were not dispatched, with a 3.5\% risk of serious adverse outcomes in a population of callers with a baseline risk of $11.1 \%$. The lower risk population constituted $16 \%$ of the cohort and around 2000 more ambulances would have been dispatched if these callers received an EMS response. As the pandemic has developed into later waves, ambulance services in the UK have come under significant sustained pressure due to increased demand. Research to improve the accuracy of EMS telephone triage, especially in terms of specificity, is needed. Due to the limitations of available routinely collected data this is likely to require robust prospective data collection. 


\section{Author Disclosure Statement}

No competing financial interests exist.

\section{Funding}

$\mathrm{CM}$ is a National Institute for Health Research (NIHR) Clinical Lecturer in Emergency Medicine (Grant Number Not Applicable/NA). This publication presents independent research funded by the National Institute for Health Research and University of Sheffield. The views expressed are those of the author(s) and not necessarily those of the University of Sheffield, the NHS, the NIHR or the Department of Health and Social Care

\section{Authors' contributions}

The idea for the study was conceived by SG, JT, TS, FB, PB and CM. Data processing and linkage was completed by TS and RC. The analyses were completed by CM and MH with specialist statistical advice from SG, JT, LS and PB. All authors contributed to interpretation of results, read and approved the final manuscript.

\section{Data sharing:}

The data used for this study are subject to data sharing agreements with NHS Digital and Yorkshire Ambulance Service which prohibits further sharing of individual level data. The data sets used are obtainable from these organisations subject to necessary authorisations and approvals.

\section{Figures:}

Figure 1: STROBE flow diagram of selection of study population

\section{References:}

1. https://www.hsj.co.uk/emergency-care/thousands-of-londons-999-calls-answered-by-otherregions/7029302.article.

2. https://www.nationalleadership.gov.uk/supporting-the-nhs-in-its-hour-of-need/londonambulance-service/.

3. Jensen $T$, Holgersen MG, Jespersen MS, et al. Strategies to Handle Increased Demand in the COVID-19 Crisis: A Coronavirus EMS Support Track and a Web-Based Self-Triage System. Prehospital Emergency Care. 2021;25:28-38.

4. Snooks H, Watkins AJ, Bell F, et al. Call volume, triage outcomes, and protocols during the first wave of the COVID-19 pandemic in the United Kingdom: Results of a national survey. Journal of the American College of Emergency Physicians Open. 2021;2:e12492. 
medRxiv preprint doi: https://doi.org/10.1101/2021.10.12.21264136; this version posted October 19, 2021. The copyright holder for this preprint (which was not certified by peer review) is the author/funder, who has granted medRxiv a license to display the preprint in perpetuity.

It is made available under a CC-BY 4.0 International license .

5. Ferron R, Agarwal G, Cooper R, Munkley D. The effect of COVID-19 on emergency medical service call volumes and patient acuity: a cross-sectional study in Niagara, Ontario. BMC Emergency Medicine. 2021;21:39.

6. Charlton K, Limmer M, Moore H. Incidence of emergency calls and out-of-hospital cardiac arrest deaths during the COVID-19 pandemic: findings from a cross-sectional study in a UK ambulance service. Emergency Medicine Journal. 2021;38:446.

7. Blackwell TH, Kaufman JS. Response time effectiveness: comparison of response time and survival in an urban emergency medical services system. Academic Emergency Medicine. 2002;9:288-295.

8. https://www.london.gov.uk/press-releases/mayoral/hospitals-at-risk-of-beingoverwhelmed-in-capital.

9. https://www.gov.uk/government/publications/covid-19-guidance-for-ambulancetrusts/covid-19-guidance-for-ambulance-trusts.

10. https://www.emergencydispatch.org/in-the-news/covid-19-communications/a332c5d1Odbf-4354-9a64-8d149f75f15d.

11. Tankel JW, Ratcliffe D, Smith M, et al. Consequences of the emergency response to COVID19: a whole health care system review in a single city in the United Kingdom. BMC Emergency Medicine. 2021;21:55.

12. https://digital.nhs.uk/services/national-data-opt-out.

13. Campbell MJ, Jacques RM, Fotheringham J, Maheswaran R, Nicholl J. Developing a summary hospital mortality index: retrospective analysis in English hospitals over five years. BMJ. 2012;344:e1001.

14. Street A, Maynou L, Gilbert T, Stone T, Mason S, Conroy S. The use of linked routine data to optimise calculation of the Hospital Frailty Risk Score on the basis of previous hospital admissions: a retrospective observational cohort study. The Lancet Healthy Longevity. 2021;2:e154-e162.

15. Rockwood K, Song $X$, MacKnight $C$, et al. A global clinical measure of fitness and frailty in elderly people. CMAJ. 2005;173:489-495.

16. Hirst E, Irving A, Goodacre S. Patient and public involvement in emergency care research. Emergency Medicine Journal. 2016;33:665-670.

17. https://digital.nhs.uk/binaries/content/assets/website-assets/publications/publicationsadmin-pages/related-documents/disclosure-control-procedure.pdf.

18. Marincowitz $C$, Stone $T$, Bath $\mathrm{P}$, et al. Accuracy of telephone triage for predicting adverse outcome in suspected COVID-19: An observational cohort study. medRxiv.

2021:2021.2006.2024.21259441.

19. Goodacre S, Thomas B, Lee E, et al. Characterisation of 22445 patients attending UK emergency departments with suspected COVID-19 infection: Observational cohort study. PLOS ONE. 2020;15:e0240206.

20. https://digital.nhs.uk/data-and-information/publications/statistical/health-survey-forengland/2019.

21. Zachariasse JM, van der Hagen V, Seiger N, Mackway-Jones $K$, van Veen M, Moll HA. Performance of triage systems in emergency care: a systematic review and meta-analysis. BMJ Open. 2019;9:e026471.

22. Thomas B, Goodacre $S$, Lee $E$, et al. Prognostic accuracy of emergency department triage tools for adults with suspected COVID-19: the PRIEST observational cohort study. Emergency Medicine Journal. 2021:emermed-2020-210783.

23. https://www.yorkpress.co.uk/news/19012031.yorkshire-ambulance-service-declares-majorincident---report/.

24. https://www.reuters.com/article/us-health-coronavirus-britain-manchester-idUKKBN27I2HK. 25. https://cdn.emergencydispatch.org/iaed/pdf/NAE-Protocol-36-Flu-v13_3.pdf. 
medRxiv preprint doi: https://doi.org/10.1101/2021.10.12.21264136; this version posted October 19, 2021. The copyright holder for this preprint (which was not certified by peer review) is the author/funder, who has granted medRxiv a license to display the preprint in perpetuity.

It is made available under a CC-BY 4.0 International license.

26. Goodacre S, Thomas B, Sutton L, et al. Derivation and validation of a clinical severity score for acutely ill adults with suspected COVID-19: The PRIEST observational cohort study. PLOS One. 2021;16:e0245840.

27. Abbasi J. Study Suggests Lasting Immunity After COVID-19, With a Big Boost From Vaccination. JAMA. 2021;326:376-377. 